\title{
Effects of Transthoracic Echocardiography on the Prognosis of Patients with Acute Respiratory Distress Syndrome: A Propensity Score Matched Analysis of the MIMIC-III Database
}

\author{
Daoran Dong \\ Shaanxi Provincial People's Hospital \\ Yan Wang \\ Shaanxi Provincial People's Hospital \\ Chan Wang \\ Shaanxi Provincial People's Hospital \\ Yuan Zong ( $\nabla$ spphicu@163.com ) \\ Shaanxi Provincial People's Hospital
}

Research Article

Keywords:

Posted Date: January 12th, 2022

DOI: https://doi.org/10.21203/rs.3.rs-1169012/v2

License: (c) (i) This work is licensed under a Creative Commons Attribution 4.0 International License. Read Full License 


\section{Abstract}

Introduction: Acute respiratory distress syndrome (ARDS) has high mortality and is mainly related to the circulatory failure. Therefore, real-time monitoring of cardiac function and structural changes has important clinical significance. Transthoracic echocardiography (TTE) is a simple and noninvasive realtime cardiac examination which is widely used in intensive care unit (ICU) patients. The purpose of this study was to analyze the effect of TTE on the prognosis of ICU patients with ARDS.

Methods: The data of ARDS patients were retrieved from the MIMIC-III v1.4 database and patients were divided into the TTE group and non-TTE group. Then, the baseline data were compared between the two groups, and the effect of TTE on the prognosis of ARDS patients was analyzed through multivariate logistic analysis and the propensity score (PS).

Results: A total of 1,346 ARDS patients were enrolled, including 519 (38.6\%) cases in the TTE group and $827(61.4 \%)$ cases in the non-TTE group. Compared with the non-TTE group, the 28-day mortality of patients in the TTE group was greatly improved (OR=0.64 95\% Cl: $0.48-0.86, P=0.003)$. The length of ICU stay in the TTE group was significantly shorter than that in the non-TTE group (17d vs.14d, $P=0.0001)$. The infusion volume in the TTE group was significantly less than that of the non-TTE group (6.2L vs.5.5L on day $1, P=0.0012)$. Importantly, the patients in the TTE group were weaned ventilators earlier than those in the non-TTE group (ventilator-free days within $28 \mathrm{~d}$ : $21 \mathrm{~d} v s .19 .8 \mathrm{~d}$, respectively, $\mathrm{P}=0.071$ ).

Conclusion: TTE can lower the risk of 28-d mortality in patients with ARDS.

\section{Introduction}

Acute respiratory distress syndrome (ARDS) is associated with adverse clinical outcomes and has an approximate overall mortality rate of $40 \%$, despite the most standard treatment. ARDS patients have been shown to have a good tolerance to relative hypoxemia. However, their survival rate is not necessarily increased by the improvement of oxygenation[1]. ARDS patients frequently suffer from circulatory failure, which is independently related to death[2] and often accompanied by hemodynamic instability, such that more than $60 \%$ of the patients suffer from hemodynamic disorders, among which $65 \%$ need to use catecholamine drug[3, 4]. ARDS causes shock according to the following three factors: (1) pulmonary arterial pressure (PAP) elevation caused by vasoconstriction due to microthrombosis, arterial remodeling and hypoxia, acidosis and/or inflammatory mediators; (2) the impact of mechanical ventilation on the function of the right ventricle (RV); (3) RV failure caused by abnormal tissue oxygen demand and hemodynamic disorders due to sepsis, which probably results from insufficient preload or excessive afterload. Assessing the volume status and correcting hemodynamic disorders helps to avoid the second attack based on hypoxemia and represents an important part of the ARDS treatment[5].

Hemodynamic monitoring of ARDS patients contributes to developing reasonable therapeutic regimens and improving the prognosis. However, both Computer Tomography(CT) and chest X-ray suffer from deficiencies in the diagnosis and prognostic evaluation of ARDS[6]. The rapid progress in ultrasonic 
techniques for critical diseases has made it possible to achieve early diagnosis and prognostic evaluation of ARDS patients. Doctors can judge the disease severity and promptly adjust the treatment plan with the help of transthoracic echocardiography (TTE).

Despite these advances, the rationality of TTE for ARDS patients has not yet been assessed, and active adjustment of the treatment plan based on the results of ultrasonography is not always achieved by doctors[7]. In addition, preoperative echocardiography was found unable to reduce mortality and the length of stay for patients undergoing non-cardiac surgery[8]. Some studies also suggested that TTE cannot improve the Acute Physiology and Chronic Health Evaluation II(APACHEII) score for the risk of death in critically ill patients[9].

In this study, we perform a retrospective analysis to observe whether TTE can affect the short-term prognosis and related indexes of intensive care unit (ICU) patients with ARDS. This work provides a definite basis for the rational use of TTE in clinical practice, reducing the overuse of it and optimizing the allocation of medical resources.

\section{Methods}

\section{Study population}

The data in this study came from the MIMIC-III database. MIMIC-III is an open access medical database, jointly released by the Massachusetts Institute of Technology(MIT) Laboratory for Computational Physiology, Beth Israel Deaconess Medical Center, Philips Medicine and the National Institutes of Health[10]. It contains the hospitalization information of more than 50,000 patients admitted to Beth Israel Deaconess Medical Center from June 2001 to October 2012, including the vital signs, drugs, laboratory test results, clinical observation results, records made by nurses, fluid balance, imaging reports, length of stay and survival data.

All the patients in the database were screened. Inclusion criteria were as follows: (1) adults aged $\geq 18$ years old, with complete medical records, including ultrasound reports during the ICU stay; (2) length of ICU stay $\geq 72 \mathrm{~h}$; (3) meeting the Berlin criteria for ARDS[11]. ARDS is defined as follows: acute attack, oxygenation index $\leq 300 \mathrm{mmHg}$, bilateral infiltration on chest $X$-ray and no heart failure. Based on the oxygenation index, ARDS is classified into mild, moderate and severe degrees. For patients admitted to the ICU several times, only the data related to the first ICU admission were considered.

\section{Data extraction}

Rather than the assessment of volume and cardiac function in ARDS patients, echocardiography can also be used for the routine assessment of ICU admission. Thus, only the patients who underwent echocardiography within $24 \mathrm{~h}$ of mechanical ventilation were enrolled in the study. The enrolled ARDS patients were divided into Echo_1 group and non-Echo_1 group according to whether echocardiography was performed within $24 \mathrm{~h}$ of mechanical ventilation. In addition, in order to assess the timing of echocardiography, the patients were also divided into the Echo_2 group and non-Echo_2 group according 
to whether echocardiography was performed within $24 \mathrm{~h}$ after shock (Fig. 1). The variables we extracted or calculated including the baseline characteristics, comorbidities, vital signs within 24 hours after entering the ICU, and parameters on the first day of mechanical ventilation. The first 24 hours after entering the ICU (ie the baseline value) and the extreme values during the ICU stay [ie the maximum and minimum values]. All patients were evaluated for the severity score of organ dysfunction within 24 hours after admission. The details of collected data are listed in Table S1.

The value of lactic acid was not collected in more than $30 \%$ of patients in this cohort. Thus, using it directly as a covariate would result in a large number of missing values. Therefore, it was used as a covariate for stratification.

\section{Outcome indexes}

The primary outcome in this study was the 28-d post-admission mortality rate. The secondary outcomes included the ventilator- and vasopressor-free days within $28 \mathrm{~d}$ after ICU admission and total intravenous infusion volume on days 1,2 and 3 of the mechanical ventilation.

\section{Statistical methods}

The data were expressed as mean \pm standard deviation, median of interquartile range and ratio (absolute and relative frequency) according to the actual conditions. Continuous data was assessed for normal distribution by Skewness-kurtosis test (sktest) and compared using the Student's $t$ test or Mann-Whitney test, while categorical variables were compared using the $\chi^{2}$ test or Fisher's exact test. Statistical analyses were conducted using Stata (version 15.0, StataCorp, College Station, Texas). The propensity score (PS) of patients undergoing TTE was assessed using the psestimate command to minimize the imbalance of variables between the TTE and non-TTE groups[12]. With PS as the weight, the weighted queue was generated by the inverse probability weighting (IPW) model. A P $<0.05$ was considered to be statistically significant[13].

Multivariate logistic regression was conducted on the correlation between echocardiography and mortality after matching to assess the independent impact of echocardiography on the prognosis of ARDS patients.

\section{Results}

Among the 46,476 ICU patients with 61,532 data elements (times of ICU admission) in the MIMIC-III v1.4 database, the Berlin criteria were met in 2,224 elements, and 1,346 patients were finally enrolled in the study and divided into the Echo_1 group: 519 (38.56\%) cases, including 219 women (42.2\%), and nonEcho_1 group: 827 (61.44\%) cases, including 334 women (40.4\%). The characteristics of this cohort are shown in Table 2. The simplified acute physiology score(SAPS) and Sequential Organ Failure Assessment(SOFA) scores were higher in the Echo_1 group than those in non-Echo_1 group (SAPS: 44 points vs. 40 points, respectively, $\mathrm{P}<0.001$ ) (SOFA: 7 points vs. 6 points, respectively, $\mathrm{P}<0.001$ ). Besides, the proportions of patients receiving mechanical ventilation (MV) and using vasoactive drugs (VD) were significantly higher in Echo_1 group than those in non-Echo_1 group (MV: 89.4\% vs. 84.0\% respectively) 
(VD: $51.6 \%$ vs. $42.8 \%$ respectively). It can be seen that the patients undergoing TTE have severer disease than those without TTE (see Table 2 for details). Before PS matching, there were differences in the following parameters between the Echo_1 and non-Echo_1 groups: oxygenation index, positive end expiratory pressure(PEEP), systolic blood pressure, mean arterial pressure(MAP), plateau pressure, $\mathrm{Ca}^{2+}$, $\mathrm{pH}$, age, SOFA score, SAPSII, Oxford acute severity of illness score (OASIS), respiratory rate, ARDS severity, ICU type, admission type and the presence or absence of sepsis (Table 2). After PS matching (1:1) between 440 patients undergoing echocardiography and 440 patients without echocardiography, the imbalance between the Echo_1 and non-Echo_1 groups was significantly reduced (Fig. S1), and all baseline variables were comparable between the two groups (Table 2). After matching, the 28-d mortality rate significantly declined in the Echo_1 group compared with the non-Echo_1 group $(25.9 \%$ vs. $35.2 \%$, respectively, $\mathrm{P}=$ $0.003)$, and the adjusted $\mathrm{OR}$ value was $0.64(95 \% \mathrm{Cl}$ : $0.48-0.86, \mathrm{P}=0.003)$. The analysis results of secondary endpoints revealed more ventilator- and vasopressor-free days in the TTE group compared with the non-TTE group. The infusion volume in the TTE group was significantly smaller than that in non-TTE group on day $1(6.1 \mathrm{~L}$ vs. $5.5 \mathrm{~L}$, respectively, $\mathrm{P}=0.028)$ and day $3(3.2 \mathrm{~L} v$ s. $2.7 \mathrm{~L}$, respectively, $\mathrm{P}=0.008)$. The TTE group had a significantly shorter time of using vasopressor and ventilator compared with the nonTTE group (vasopressor-free days within $28 \mathrm{~d}$ : $26.8 \mathrm{~d}$ vs. $27.3 \mathrm{~d}$, respectively, $\mathrm{P}=0.033$, ventilator-free days within $28 \mathrm{~d}$ : $17 \mathrm{~d}$ vs. $19.8 \mathrm{~d}$, respectively, $\mathrm{P}=0.0202$ ). No statistically significant differences were observed in other secondary endpoints between the two groups (Table 3).

Before PS matching, there were differences between the Echo_2 group and non-Echo_2 group in the following parameters: the oxygenation index, PEEP, systolic blood pressure, MAP, plateau pressure, peak airway pressure, blood urea nitrogen(BUN), weight, minute volume, pH, SOFA score, SAPSII, OASIS, respiratory rate, ARDS severity, ICU type, lactic acid and the presence or absence of sepsis (Fig. S1). After PS matching (1:1) between 314 patients undergoing echocardiography and 316 patients without echocardiography, the imbalance between the Echo_2 and non-Echo_2 groups was significantly reduced and all baseline variables were comparable between the two groups (Fig. S1). After matching, there was no significant difference in the 28-d mortality rate between the Echo_2 and non-Echo_2 groups (32.9\% vs. $29.9 \%$, respectively, $\mathrm{P}=0.418)$, and the adjusted $\mathrm{OR}$ value was $0.87(95 \% \mathrm{Cl}: 0.62-1.22, \mathrm{P}=0.418)$. The analysis results of secondary endpoints revealed a significantly shorter length of stay in the TTE group than that in the non-TTE group ( $12.5 \mathrm{~d}$ vs. $11 \mathrm{~d}$, respectively, $\mathrm{P}=0.017$ ), while other secondary endpoints showed no statistically significant differences between the two groups (Table 4).

\section{Discussion}

ARDS is a life-threatening pulmonary disease with a poor prognosis and an increased mortality rate[14, 15]. Such an adverse outcome may result from circulatory failure rather than hypoxemia[2]. Critical ultrasound examination is a rapid, non-invasive and reproducible operation, with a dynamic and visual presentation of the results, combining the monitoring results and diagnosis and treatment thoughts for critical disease. It plays an increasingly important role in the clinical diagnosis and treatment of ARDS. 
Previous studies mainly focused on its diagnosis and treatment of ARDS, while the influencing factors for its prognosis and the value of ultrasonography for the prognostic evaluation have rarely been studied. It is of great significance to determine the actual application value of the auxiliary examination for the patients. First, there should be good reasoning for the patients requiring examination. Second, unnecessary examination and occupation of the medical resources should be avoided to ease the economic burden on the individuals, society and country. Therefore, continuous testing in clinical practice is needed for any new technique to determine its application value. The MIMIC-III database, established based on the electronic medical record, is a continuously updated dynamic data system, which reflects the diagnosis and treatment process of critically ill patients and has been commonly used by the researchers of intensive care medicine[16].

To the best of our knowledge, this is the first study assessing the effect of echocardiography on the prognosis of ARDS patients. In this work, patients undergoing TTE had a higher disease severity score and more comorbid conditions, suggesting a severer disease degree. It was found that the 28-d mortality rate of patients undergoing TTE significantly declined after adjusting the confounding factors. Several hypotheses were proposed to explain the survival benefit, and some variables were compared between the TTE and non-TTE groups. The infusion volume in the TTE group was smaller at day 1 and day 3 after ventilation. Vasopressors were used more often in the TTE group, probably because TTE promotes the timely use of vasopressors. Patients in the TTE group also stopped taking vasopressors earlier than those in non-TTE group, which could be related to several factors according to the analysis of PS matching. First, studies have shown that acute pulmonary heart disease occurs in 20-25\% of ARDS patients[17], and many obstructive factors, including hypoxia-induced pulmonary vasospasm, hypercapnia, high airway pressure, inflammatory factor-induced vasoconstriction and lung volume collapse cause an increased pulmonary vascular resistance, which greatly affects the right heart function and pulmonary circulatory resistance[18]. During positive pressure ventilation, pulmonary vessels are compressed by the stretched alveoli, which leads to increased pulmonary artery resistance and obviously reduces the pulmonary circulation blood flow[19]. The increase in PEEP raises the pulmonary vascular resistance, which leads to right heart dysfunction and may eventually result in the occurrence and development of shock.

Echocardiography assessment of the right heart function during treatment can reveal the major cause of hemodynamic involvement or instability, because the status of the right heart involvement greatly varies among different types of shock, which directly affects the development and implementation of the clinical therapeutic regimen. Second, in terms of fluid management, right heart enlargement can be caused by an acute increase in the blood volume. Acute right heart enlargement occurs when there is a failure of compensatory fluid discharge due to renal insufficiency or low MAP. This results in left ventricular diastolic restriction through the ventricular septum and the pericardium, increasing the left ventricular filling pressure, and thus the extravascular lung water[20].The fluid management strategy test of the ARDS Collaboration in 2006 well established that patients with ARDS can mostly benefit from the conservative fluid management strategy through shock correction (vasopressor dependence), keeping the circulatory stability and guaranteeing organ perfusion. Although the conservative strategy does not reduce the 60-d mortality, it can shorten the duration of mechanical ventilation and length of ICU stay and ameliorate oxygenation without increasing the incidence of other organ dysfunction[21]. Moreover, a systematic 
review and meta-analysis covering 2051 patients with sepsis and/or ARDS in 11 randomized trials in 2017 found no significant difference in the mortality rate between the restricted fluid management group and routine treatment group[22]. However, the ventilator-free duration was found to be significantly increased and the length of ICU stay was significantly shortened in the restricted fluid management group. Our study showed that the infusion volume was smaller in the TTE group, which may have contributed to the improved survival. Third, vasoactive drugs are an important treatment means to lower the pulmonary circulatory pressure. As the distribution of related receptors varies, attention should be paid to the different effects of vasoactive drugs on pulmonary circulation and systemic circulation. Generally, vasodilators may also affect the systemic circulation when they act on the pulmonary circulation, a contradiction that may be sharper in severely ill patients. On the one hand, vasodilators expand the pulmonary artery and lower the pulmonary circulatory resistance, which supports the restoration of the right heart function and reduces the central venous pressure (CVP). On the other hand, vasodilators reduce the systemic circulation pressure, leading to circulatory instability. In particular, when less obvious decline in PAP and obvious decline in the systemic circulation pressure are observed when vasodilators are used, the transseptal pressure will be altered, which results in a leftward shift of the interventricular septum, a significant decrease in the left ventricular end-diastolic volume and a decline in the cardiac output. As a result, the systemic circulation pressure is further decreased, leading to an autonomous vicious cycle of the right heart[23]. It has been confirmed that these drugs can be applied under the guidance of tricuspid annular plane systolic excursion(TAPSE), right/left ventricular area ratio and eccentricity index[17]. The abovementioned discussion may include the reasons for the improved mortality of ARDS patients by ultrasonography.

In addition, the patients were divided into the Echo_2 group and non-Echo_2 group according to the time of ultrasonography. Unlike the Echo_1 group, patients in the Echo_2 group showed no improvement regarding the clinical outcome, with no significant difference in the 28-d mortality between the Echo_2 group and control group. It is believed that ultrasonography should be performed as soon as possible on ARDS patients to reduce the mortality rate to the largest extent. However, no relevant studies have been presented. Hence, larger-scale prospective randomized controlled trials should be performed in the future to determine the timing of ultrasonography for ARDS patients. Although early assessment will not necessarily help to avoid further lung injury throughout the course of the disease, it can enable us to adjust the ventilation strategy, thereby ameliorating the prognosis. Based on our experience, we can suggest that ALI or ARDS patients receive ultrasonography at the time of ICU admission, and regular review should be performed according to the disease condition. Echocardiography at admission can provide valuable information, not only about the current clinical conditions, but also about preexisting diseases (e.g., severe right ventricular hypertrophy suggests the presence of chronic lung disease). If the condition is stable, then echocardiography should be performed at least once a week before weaning and after extubation (i.e., fluid therapy monitoring). Moreover, echocardiographic can assist in determining the cause (or auxiliary factor) of the progressive respiratory failure, such as systolic pulmonary artery pressure(sPAP) elevation, right ventricular dilatation, progressive right or left ventricular failure or individualized treatment. Reexamination (ventilation and non-ventilation) should be performed whenever right ventricular dilation or dysfunction is developed (even by conventional echocardiography), other options (i.e., inhalation of nitric 
oxide and prone position) should also be considered, and patients should be monitored more closely using echocardiography.

Our study had some limitations. First, despite the large sample size of real data, the data came from a single medical center, and there may be deviations among the subjects in the medical level, habits and population. Second, this was a retrospective analysis, so a large amount of data might have been eliminated, and there might have been a selection bias due to the lack of key information and other reasons during data extraction. Although TTE is a non-invasive and convenient operation, it has a poor repeatability. We could not assess the consistency of TTE in this study, resulting in a measurement bias. Therefore, we suggest designing a prospective multi-center study based on similar studies to further observe the effects of TTE on patients with severe ARDS. Third, although the 28-d mortality rate was explored, some significant outcome variables were not considered in the analysis, including long-term mortality and ICU readmission. Finally, the MIMIC-III database included the cases before 2012. Some studies suggest expanding the Berlin criteria for ARDS to include patients who have undergone high-flow nasal oxygen therapy (at least $30 \mathrm{~L} / \mathrm{min}$ ) and meet other standards in the Berlin criteria[24], which may lead to a result deviation in this study.

\section{Conclusion}

TTE can lower the 28-d mortality rate of critically ill ARDS patients. Its mechanism remains to be explored, but under the guidance of the TTE results, we suggest that it may be related to the evaluation of right heart function and adjustment of fluid and vasoactive drugs. In future work, more large-scale prospective studies are needed to explore the influencing mechanism of ultrasound to the long-term prognosis of patients with ARDS and the timing of ultrasound use.

\section{Abbreviations}

MIMIC-III

The medical information mart for intensive care III

ARDS

Acute Respiratory Distress Syndrome

TTE

Transthoracic echocardiography

ICU

Intensive care unit

PS

Propensity score

OR

Odds ratio

PAP

Pulmonary arterial pressure 
RV

Right ventricle

CT

Computer Tomography

APACHEII

Acute Physiology and Chronic Health Evaluation II

MIT

Massachusetts Institute of Technology

IPW

Inverse probability weighting

SAPS

Simplified acute physiology score

SOFA

Sequential Organ Failure Assessment

MV

Mechanical ventilation

VD

Vasoactive drugs

PEEP

Positive end expiratory pressure

MAP

Mean arterial pressure

OASIS

Oxford acute severity of illness score

BUN

Blood urea nitrogen

CVP

Central venous pressure

TAPSE

Tricuspid annular plane systolic excursion

SPAP

systolic Pulmonary artery pressure

$\mathrm{CHF}$

Congestive heart failure

AFIB

Atrial fibrillation

CKD

chronic kidney disease

COPD

chronic obstructive pulmonary disease 
CAD

coronary artery disease

$\mathrm{SpO} 2$

pulse oxygen saturation

$\mathrm{PO} 2$

oxygen partial pressure

$\mathrm{PCO} 2$

carbon dioxide partial pressure.

\section{Declarations}

\section{Ethics approval and consent to participate}

The establishment of this database was approved by the Massachusetts

Institute of Technology (Cambridge, MA) and Beth Israel Deaconess Medical Center (Boston, MA), and consent was obtained for the original data collection. Therefore, the ethical approval statement and the need for informed consent were waived for this manuscript

\section{Consent for publication}

Not applicable.

\section{Competing interests}

The authors declare that they have no competing interests.

\section{Acknowledgements}

We would like to thank the Massachusetts Institute of Technology and the Beth Israel Deaconess Medical Center for the MIMIC project.

\section{Authors' contributions}

DDR and ZY conceptualized the research aims, planned the analyses, and guided the literature review. DDR extracted the data from the MIMIC-IV database. DDR, WC and WY participated in data analysis and interpretation. DDR wrote the first draft of the paper and the other authors provided comments and approved the final manuscript.

\section{Funding}

This research was funded by Shaanxi Provincal key research and development project "The Application of Right Heart Protective Ventilation Strategy in Patients with Acute Respiratory Distress Syndrome" (Project Number: 2021SF-257). 
Availability of data and materials

The datasets analysed during the current study are available in the MIMIC-III repository, https://physionet.org/content/mimiciv/0.4/.

\section{References}

1. Mehdi Mezidi CG.Conservative versus liberal oxygenation targets for mechanically ventilated patientsa pilot multicenter randomized controlled trial. J Thorac Dis 2016, 8(3):307-310.

2. Vieillard-Baron A GE, Valente E.Predictors of mortality in acute respiratory distress syndrome. Focus On the role of right heart catheterization. Am J Respir Crit Care Med 2000, 161:1597-1601.

3. McAuley DF LJ, O'Kane CM. Simvastatin in the Acute Respiratory Distress Syndrome. N Eng/ J Med 2014, 371:1695-1703.

4. Charron $\mathrm{C} B K$, Caille V. Routine prone positioning in patients with severe ARDS feasibility and impact on prognosis. Intensive Care Med 2011, 37:785-790.

5. Repesse X CC, Vieillard-Baron A. Right ventricular failure in acute lung injury and acute respiratory distress syndrome. Minerva Anestesiologica 2012, 78(8)::941-948.

6. O BTGSM. The standard of care of patients with ARDS ventilatory settings and rescue therapies for refractory hypoxemia. Intensive Care Med 2016, 42(5):699-711.

7. Matulevicius SA RA, Das SR. Appropriate use and clinical impact of transthoracic echocardiography. JAMA Intern Med 2013, 173:1600-1607.

8. Wijeysundera DN BW, Karkouti K. Association of echocardiography before major elective non-cardiac surgery with postoperative survival and length of hospital stay population based cohort study. BMJ 2011, 342:d3695.

9. Sawchuk CW WD, Kavanagh BP. Transthoracic echocardiography does not improve prediction of outcome over APACHE II in medical-surgical intensive care. Can J Anaesth 2003, 50:305-310.

10. Johnson AE PT, Shen L. MIMIC-III, a freely accessible critical care database. Sci Data 2016, 3:160035.

11. Ranieri VM RG, Thompson BT. Acute respiratory distress syndrome the Berlin Definition. JAMA 2012, 307:2526-2533.

12. Imbens GW. Matching Methods in Practice Three Examples. The Journal of Human Resources 2014, 50(2):373-418.

13. Cole SR HM. Constructing inverse probability weights for marginal structural models. Am J Epidemiol 2008, 168:656-664.

14. Bellani G LJ, Pham T. Epidemiology, Patterns of Care, and Mortality for Patients With Acute Respiratory Distress Syndrome in Intensive Care Units in 50 Countries. JAMA 2016, 315:788-800.

15. Herridge MS TC, Matte A. Functional disability 5 years after acute respiratory distress syndrome. $N$ Engl J Med 2011, 364:1293-1304. 
16. Weeks HL BC, McNeer E. medExtractR A targeted, customizable approach to medication extraction from electronic health records. J Am Med Inform Assoc 2020, 27:407-418.

17. Sundar Krishnan GAS. Acute right ventricular dysfunction real-time management with echocardiography. CHEST 2015, 147(3):835 - 846.

18. Greyson CR. Pathophysiology of right ventricular failure. Crit Gare Med 2008, 36(Suppl.):S57-S65.

19. Francois Jardin AVB. Right ventricular function and positive pressure ventilation in clinical practice from hemodynamic subsets to respirator settings. Intensive Care Med 2003, 29(9):1426-1434.

20. Jamie R. Mitchell WAW, Rozsa Sas,et al. RV filling modulates LV function by direct ventricular interaction during mechanical ventilation. Am J Physiol Heart Circ Physiol 2005, 289(2):H549-557.

21. The National Heart L, and Blood Institute Acute Respiratory Distress Syndrome (ARDS) Clinical Trials Network. Comparison of Two Fluid-Management Strategies in Acute Lung Injury. N Engl J Med 2006, 354(24):2564-2575.

22. Jonathan A. Silversides EM, Andrew J. Ferguson, et al. Conservative fluid management or deresuscitation for patients with sepsis or acute respiratory distress syndrome following the resuscitation phase of critical illness: a systematic review and meta-analysis. Intensive Care Med 2017, 43.155-170.

23. Carmen Hrymak JS, Eric Jacobsohn. Acute RV failure in ICU: Assessment and management. Can J Cardiol 2017, 33(1):61-71.

24. Michael A Matthay BTT, Lorraine B Ware. The Berlin definition of acute respiratory distress syndrome: should patients receiving high-flow nasal oxygen be included? Lancet Respir Med 2021, 9: 933-936.

\section{Tables}

Table 1 Baseline characteristics of Echo_1 before and after propensity-score matching 


\begin{tabular}{|c|c|c|c|c|c|c|}
\hline Characteristic & Before matcl & & & After matchi & & \\
\hline & $\begin{array}{l}\text { Without } \\
\text { Echo_1 }\end{array}$ & Echo_1 & $\begin{array}{l}P \\
\text { value }\end{array}$ & $\begin{array}{l}\text { Without } \\
\text { echo_1 }\end{array}$ & Echo_1 & $\begin{array}{l}\mathrm{P} \\
\text { value }\end{array}$ \\
\hline $\begin{array}{l}\mathrm{PaO} 2 / \mathrm{FiO}^{2} \\
\text { ratio(mmHg) }\end{array}$ & $139.5 \pm 63.3$ & $128.1 \pm 62.8$ & 0.001 & $130.7 \pm 61.7$ & $129.5 \pm 62.2$ & 0.779 \\
\hline PEEP( $\mathrm{cmH} 2 \mathrm{O})$ & $7.7 \pm 3.7$ & $8.1 \pm 3.9$ & 0.024 & $8.1 \pm 4.0$ & $8.0 \pm 3.8$ & 0.690 \\
\hline $\begin{array}{l}\text { Systolic blood } \\
\text { pressure }(\mathrm{mmHg})\end{array}$ & $116.2 \pm 15.5$ & $113.3 \pm 14.8$ & 0.0007 & $114.5 \pm 15.2$ & $114.1 \pm 15.1$ & 0.676 \\
\hline $\begin{array}{l}\text { Diastolic blood } \\
\text { pressure }(\mathrm{mmHg})\end{array}$ & $60.0 \pm 10.2$ & $58.9 \pm 9.1$ & 0.050 & $59.1 \pm 10.1$ & $59.4 \pm 9.3$ & 0.647 \\
\hline $\mathrm{MAP}(\mathrm{mmHg})$ & $77.7 \pm 10.3$ & $76.3 \pm 9.6$ & 0.009 & $76.6 \pm 10.2$ & $76.8 \pm 9.7$ & 0.797 \\
\hline Temperature $\left({ }^{\circ} \mathrm{C}\right)$ & $37.1 \pm 0.7$ & $37.1 \pm 0.7$ & 0.782 & $37.1 \pm 0.8$ & $37.1 \pm 0.7$ & 0.523 \\
\hline SPO2 & $96.8 \pm 2.9$ & $96.4 \pm 3.7$ & 0.049 & $96.7 \pm 3.4$ & $96.4 \pm 3.8$ & 0.355 \\
\hline $\begin{array}{l}\text { Plateau pressure } \\
(\mathrm{cmH} 2 \mathrm{O})\end{array}$ & $25.8 \pm 6.8$ & $26.9 \pm 6.9$ & 0.006 & $26.8 \pm 7.0$ & $26.6 \pm 6.8$ & 0.624 \\
\hline
\end{tabular}

$\begin{array}{lllllll}\begin{array}{l}\text { Tidal volume } \\ (\mathrm{ml} / \mathrm{kg} \text { PBW })\end{array} & 673.7 \pm 353.3 & 652.4 \pm 159.5 & 0.197 & 647.1 \pm 132.4 & 656.1 \pm 164.0 & 0.371\end{array}$

\begin{tabular}{lcccccc}
\hline $\begin{array}{l}\text { Peak inspiratory } \\
\text { pressure (cmH20) }\end{array}$ & $32.4 \pm 8.3$ & $33.2 \pm 7.9$ & 0.0600 & $33.5 \pm 8.5$ & $33.0 \pm 7.8$ & 0.312 \\
\hline $\begin{array}{l}\text { Respiratory rate } \\
\text { set }\end{array}$ & $29.7 \pm 8.4$ & $31.3 \pm 9.0$ & 0.002 & $30.8 \pm 9.2$ & $30.9 \pm 8.9$ & 0.861 \\
\hline $\begin{array}{l}\text { Blood urea } \\
\text { nitrogen (mg/dL) }\end{array}$ & $32.4 \pm 25.3$ & $34.5 \pm 26.0$ & 0.140 & $33.5 \pm 25.9$ & $33.5 \pm 25.4$ & 0.984 \\
\hline $\begin{array}{l}\text { calcium } \\
\text { (mmol/L】 }\end{array}$ & $5.5 \pm 3.3$ & $6.1 \pm 3.0$ & 0.0007 & $6.0 \pm 3.2$ & $6.0 \pm 3.1$ & 0.784 \\
& & & & & & \\
\hline PaCO2(mm Hg) & $43.9 \pm 14.0$ & $43.5 \pm 13.1$ & 0.628 & $43.6 \pm 13.3$ & $43.5 \pm 13.2$ & 0.968 \\
\hline
\end{tabular}




$\begin{array}{lllllll}\text { Arterial } \mathrm{pH} & 7.3 \pm 0.1 & 7.3 \pm 0.1 & 0.01 & 7.3 \pm 0.1 & 7.3 \pm 0.1 & 0.8423\end{array}$

Platelet $(\times 109$ /L】 $\quad 213.4 \pm 130.4 \quad 211.1 \pm 121.4 \quad 0.750 \quad 204.2 \pm 129.3 \quad 209.4 \pm 118.1 \quad 0.534$

$\begin{array}{lllllll}\begin{array}{l}\text { Potassium } \\ \text { mmol/L } \mathbb{M}\end{array} & 4.2 \pm 0.88 & 4.2 \pm 0.88 & 0.757 & 4.2 \pm 0.86 & 4.2 \pm 0.87 & 0.813\end{array}$

(mmol/L】

$\begin{array}{lllllll}\begin{array}{l}\text { Creatinine } \\ (\mu \mathrm{mol} / \mathrm{L} \rrbracket\end{array} & 1.5 \pm 1.4 & 1.5 \pm 1.3 & 0.578 & 1.4 \pm 1.2 & 1.4 \pm 1.2 & 0.847\end{array}$

$\begin{array}{lllllll}\text { Sodium }(\mathrm{mmol} / \mathrm{L} \bigotimes & 138.7 \pm 5.3 & 138.7 \pm 5.6 & 0.804 & 139.0 \pm 5.0 & 138.6 \pm 5.7 & 0.212\end{array}$

\begin{tabular}{|c|c|c|c|c|c|c|}
\hline Age & $67.9 \pm 46.0$ & $62.2 \pm 31.0$ & 0.013 & $63.5 \pm 35.0$ & $62.5 \pm 33.0$ & 0.672 \\
\hline Weight (kg) & $83.7 \pm 19.5$ & $87.0 \pm 52.3$ & 0.101 & $84.4 \pm 19.2$ & $86.6 \pm 54.9$ & 0.426 \\
\hline $\begin{array}{l}\text { Minute ventilation } \\
(\mathrm{I} / \mathrm{min})\end{array}$ & $12.9 \pm 8.8$ & $13.2 \pm 5.7$ & 0.460 & $13.0 \pm 4.8$ & $13.0 \pm 5.8$ & 0.997 \\
\hline SOFA & $6 \rrbracket 4-8 \rrbracket$ & $7 \rrbracket 5-10 \otimes$ & 0.0000 & $7(5-9.5)$ & $7(5-9.5)$ & 0.768 \\
\hline SAPS II & $40(32-50)$ & $44 \llbracket 34-54 \rrbracket$ & 0.0000 & $41(33-52.5)$ & $43(33-53)$ & 0.246 \\
\hline OASIS & $37 \rrbracket 31-43 \rrbracket$ & $39 \llbracket 34-45 \rrbracket$ & 0.0003 & $39(33-43)$ & $38.5(33-44)$ & 0.875 \\
\hline $\begin{array}{l}\text { Elixhauser } \\
\text { comorbidity score }\end{array}$ & $1 \otimes 1-12 \rrbracket$ & $6 \rrbracket 2-13 \rrbracket$ & 0.781 & $7(2-12)$ & $6(2-12.5)$ & 0.573 \\
\hline
\end{tabular}

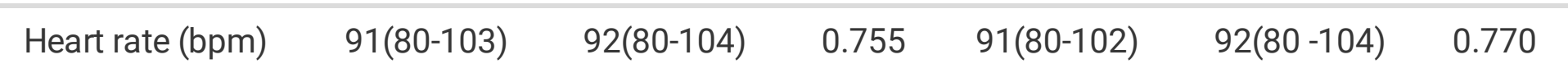

$\begin{array}{lllllll}\begin{array}{l}\text { Mean respiratory } \\ \text { rate }(/ \mathrm{min})\end{array} & 20(17-24) & 21(18-25) & 0.002 & 21(17-24) & 21(18-24) & 0.83\end{array}$




\begin{tabular}{|c|c|c|c|c|c|c|}
\hline 1 & $154(18.6 \%)$ & 77 (14.8\%) & & $64(14.5 \%)$ & $66(15 \%)$ & \\
\hline 2 & $396(47.9 \%)$ & $223(43.0 \%)$ & & $196(44.5 \%)$ & 197(44.8\%) & \\
\hline 3 & $277(33.5 \%)$ & $219(42.2 \%)$ & & $180(40.9 \%)$ & $177(40.2 \%)$ & \\
\hline ICU type & & & 0.024 & & & 0.288 \\
\hline $\mathrm{CCU}$ & $93(11.2 \%)$ & 80 ( $15.4 \%)$ & & $54(12.3 \%)$ & $67(15.2 \%)$ & \\
\hline CSRU & $140(16.9 \%)$ & $\begin{array}{l}40 \\
7.7 \%)\end{array}$ & & $50(11.4 \%)$ & $37(8.4 \%)$ & \\
\hline MICU & $362(43.8 \%)$ & $265(51.1 \%)$ & & $209(47.5 \%)$ & $224(50.9 \%)$ & \\
\hline SICU & $111(13.4 \%)$ & $67(12.9 \%)$ & & $61(13.9 \%)$ & $57(13.0 \%)$ & \\
\hline TSICU & $121(14.6 \%)$ & $67(12.9 \%)$ & & $66(15 \%)$ & $55(12.5 \%)$ & \\
\hline Admission type & & & 0.001 & & & 0.068 \\
\hline ELECTIVE & $102(12.3 \%)$ & $33(6.4 \%)$ & & $38(8.6 \%)$ & $6.6 \rrbracket 29 \rrbracket$ & \\
\hline EMERGENCY & $690(83.4 \%)$ & $468(90.2 \%)$ & & $376(85.5 \%)$ & $90.2 \bowtie 397 \rrbracket$ & \\
\hline URGENT & $35(4.2 \%)$ & $18(3.5 \%)$ & & $26(5.9 \%)$ & $14(3.2 \%)$ & \\
\hline Gender & & & 0.511 & & & 0.892 \\
\hline Male & $493(59.6 \%)$ & $300(57.8 \%)$ & & $253(57.5 \%)$ & $251(57.0 \%)$ & \\
\hline Diabetes & $195(23.6 \%)$ & $123(23.7 \%)$ & 0.960 & $102(23.2 \%)$ & $99(22.5 \%)$ & 0.810 \\
\hline Hypertension & $261(31.6 \%)$ & $144(27.7 \%)$ & 0.138 & $127(28.9 \%)$ & $124(28.2 \%)$ & 0.823 \\
\hline COPD & $129(15.4 \%)$ & $72(13.9 \%)$ & 0.387 & $61(13.7 \%)$ & 61(13.7\%) & 1.000 \\
\hline
\end{tabular}

\begin{tabular}{lllllll}
\hline Sepsis & $563(68.1 \%)$ & $392(75.5 \%)$ & 0.003 & $316(71.8 \%)$ & $321(73.0 \%)$ & 0.706 \\
\hline CHF & $306(37.0 \%)$ & $204(39.3 \%)$ & 0.396 & $174(39.5 \%)$ & $174(39.5 \%)$ & 1.000 \\
\hline AFIB & $249(30.1 \%)$ & $135(26.0 \%)$ & 0.105 & $118(26.8 \%)$ & $112(25.5 \%)$ & 0.645 \\
& & & & & & \\
\hline Renal & $76(9.2 \%)$ & $44(8.5 \%)$ & 0.655 & $38(8.6 \%)$ & $36(8.2 \%)$ & 0.808
\end{tabular}




\begin{tabular}{|c|c|c|c|c|c|c|}
\hline Liver & $62(7.5 \%)$ & $43(8.3 \%)$ & 0.600 & $40(9.1 \%)$ & $35(8.0 \%)$ & 0.546 \\
\hline CAD & 164(19.8\%) & $85(16.4 \%)$ & 0.112 & $77(17.5)$ & $76(17.3 \%) \rrbracket$ & 0.929 \\
\hline Stroke & $70(8.5 \%)$ & $42(8.1 \%)$ & 0.810 & $33(7.5 \%)$ & $34(7.7 \%)$ & 0.899 \\
\hline Malignancy & 135 (16.3\%) & $90(17.3 \%)$ & 0.626 & $72(16.4 \%)$ & 77(18.0\%) & 0.531 \\
\hline $\begin{array}{l}\text { Day of ICU } \\
\text { admission }\end{array}$ & & & 0.406 & & & 0.296 \\
\hline Monday & $140(16.9 \%)$ & $78(15.0 \%)$ & & $47(10.7 \%)$ & $62(14.1 \%)$ & \\
\hline Tuesday & $109(13.2 \%)$ & $66(12.7 \%)$ & & $88(20 \%)$ & $69(15.7 \%)$ & \\
\hline Wednesday & $128(15.5 \%)$ & $89(17.1 \%)$ & & $59(13.4 \%)$ & $58(13.2 \%)$ & \\
\hline Thursday & 114(13.8\%) & $62(11.9 \%)$ & & $63(14.3 \%)$ & $80(18.2 \%)$ & \\
\hline Friday & $140(16.9 \%)$ & $79(15.2 \%)$ & & $50(11.4 \%)$ & $52(11.8 \%)$ & \\
\hline Saturday & $98(11.9 \%)$ & $64(12.3 \%)$ & & $76(17.3 \%)$ & $66(15 \%)$ & \\
\hline Sunday & $98(11.9 \%)$ & $81(15.6 \%)$ & & $57(13.0 \%)$ & $53(12.0 \%)$ & \\
\hline Lactic acid & & & 0.119 & & & 0.395 \\
\hline Missing value & $341(41.2 \%)$ & $229(44.1 \%)$ & & $207(47.0 \%)$ & $190(43.2 \%)$ & \\
\hline$<4$ & $82(18.6 \%)$ & $64(12.3 \%)$ & & $40(10.2 \%)$ & $55(12.5 \%)$ & \\
\hline$>4$ & $404(48.9 \%)$ & 206(39.7\%) & & $188(42.7 \%)$ & $195(44.3 \%) \rrbracket$ & \\
\hline
\end{tabular}

Table 2 Outcomes of patients in matched cohort of Echo_1. 
No Echo_1 Echo_1 P value

\section{Primary outcome}

\begin{tabular}{|c|c|c|c|}
\hline 28-day mortality (n, \%) & $155 \llbracket 35.2 \% \bigotimes$ & $114 \rrbracket 25.9 \% \bigotimes$ & 0.003 \\
\hline \multicolumn{4}{|l|}{ Secondary outcomes } \\
\hline ICU mortality(n, \%) & $82 \bigotimes 18.6 \% \bigotimes$ & $76 \rrbracket 17.3 \% \bigotimes$ & 0.598 \\
\hline Length of ICU stay(d) & $11(5-19)$ & $10(5-15)$ & 0.140 \\
\hline Length of hospital stay(d) & $18 \rrbracket 10-28 \rrbracket$ & $14 \otimes 9-23 \rrbracket$ & 0.0001 \\
\hline IV fluid day 1 (mL) & $6072(3534-10235)$ & $5459(3017-8903)$ & 0.028 \\
\hline IV fluid day 2 (mL) & $3829(2036-6926)$ & 3454.877 ( $2096-6149.517)$ & 0.098 \\
\hline IV fluid day $3(\mathrm{~mL})$ & $3176(1653-6021)$ & $2666(1326-5070)$ & 0.008 \\
\hline Ventilation free days in 28 days & $17.0(0-24.7)$ & $19.8(0-25.2)$ & 0.020 \\
\hline Vasopressor free days in 28 days & $26.8(0-28)$ & $27.3(0-28)$ & 0.033 \\
\hline
\end{tabular}

Table 3 Outcomes of patients in matched cohort of Echo_2. 
No Echo_2 Echo_2 P value

Primary outcome

\begin{tabular}{|c|c|}
\hline 28-da & 104(32.9\%) \\
\hline
\end{tabular}

Secondary outcomes

ICU mortality(n, \%)

$67(21.2 \%)$

$58(17.9 \%)$

0.292

Length of ICU stay(d)

11(5-18)

$12.5(7-21)$

0.017

Length of hospital stay(d)

17(9-25.5)

18(10-29)

0.275

IV fluid day $1(\mathrm{~mL})$ 6884(4045-11061)

6945(4232-11044)

0.950

IV fluid day $2(\mathrm{~mL})$

4078(2308-6906)

4455(2586-6998)

0.470

IV fluid day $3(\mathrm{~mL})$

3606(1887 - 6960)

2910(1410-5459)

0.442

Ventilation free days in 28 days

$20(0-26)$

19(0-25)

0.111

Vasopressor free days in 28 days

$27.5(0-28)$

26.4(0-27.7)

0.0000

Table 4 isn't available with this version

\section{Figures}




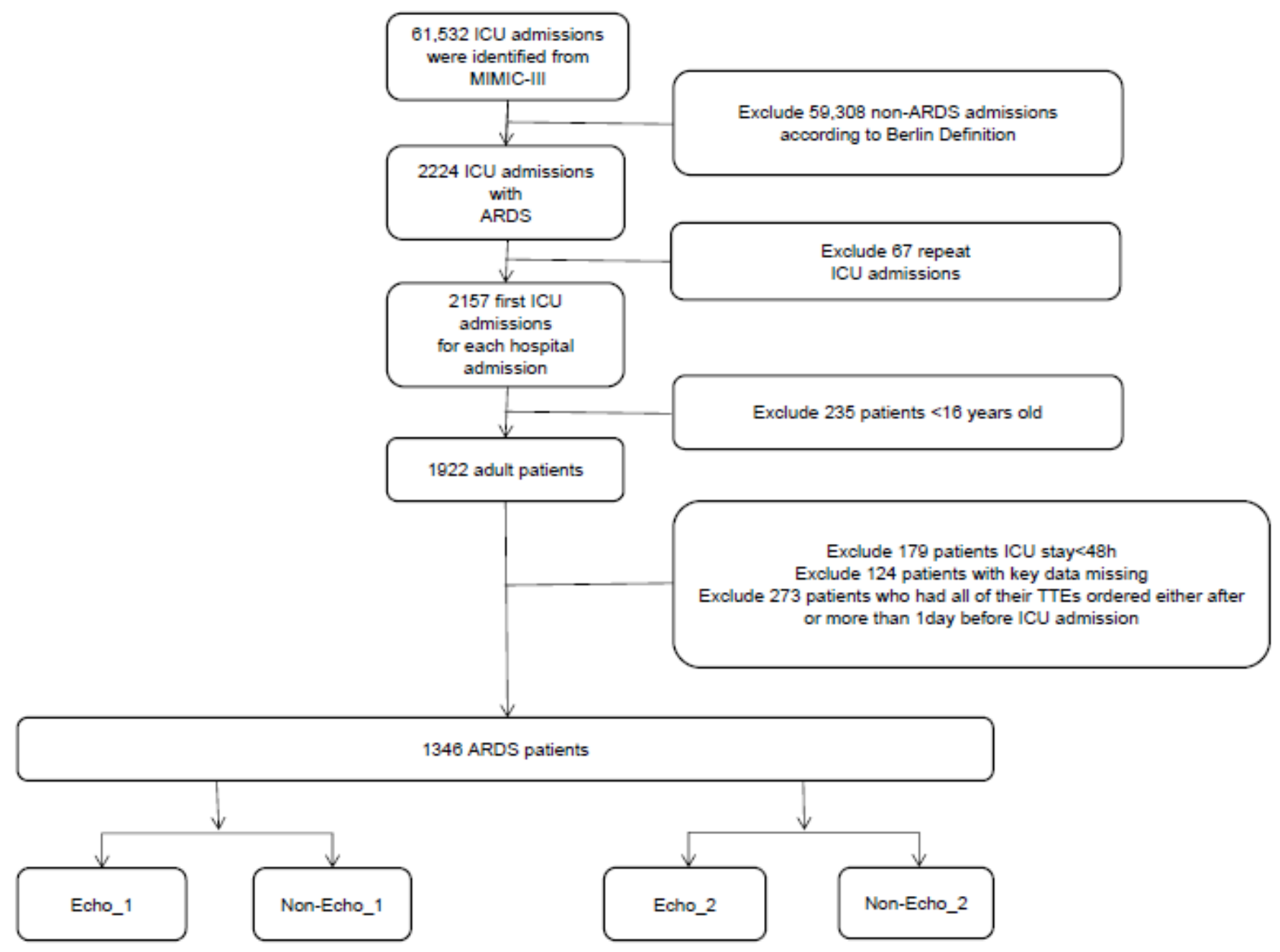

\section{Figure 1}

Flow chart of current study

\section{Supplementary Files}

This is a list of supplementary files associated with this preprint. Click to download.

- figures1Echo1propensityscorematchinggraph.pdf

- figures2Echo2propensityscorematchinggraph.pdf

- tables1.doc

- tables2.doc 